\title{
28 Research Square \\ Manifestations in Neonates Born to COVID-19 Positive Mothers: a Case Series
}

\section{Parul Jain}

Sir Ganga Ram Hospital, New Delhi

Anup Thakur ( $\nabla$ drthakuranup@gmail.com )

Sir Ganga Ram Hospital, New Delhi

Neelam Kler

Sir Ganga Ram Hospital, New Delhi

Pankaj Garg

Sir Ganga Ram Hospital, New Delhi

Case Report

Keywords: COVID-19, SARS-CoV-2, Vertical transmission

Posted Date: June 4th, 2020

DOI: https://doi.org/10.21203/rs.3.rs-32234/v1

License: (c) (i) This work is licensed under a Creative Commons Attribution 4.0 International License. Read Full License 


\section{Abstract}

Coronavirus disease (COVID-19) or severe acute respiratory syndrome coronavirus-2 (SARS-CoV-2), a novel viral infection is currently a global pandemic. Primarily transmitted by aerosols and fomites, limited information exists on its vertical transmission from mother and clinical outcomes in neonates. We report two neonates born to SARS-CoV-2 positive women.

\section{Main Text}

The world is currently battling a pandemic of a novel viral infection known as coronavirus disease (COVID-19) or severe acute respiratory syndrome coronavirus 2 (SARS-CoV-2). There is limited knowledge on vertical transmission of SARS-CoV-2 to neonates and its clinical features. We report two neonates born to SARS-CoV-2 positive women with diverse manifestations.

A 32 year old primigravida presented in labor at 38 weeks gestation. She was a booked case, with no significant medical history and uneventful antenatal course. An emergency C-section was contemplated in view of non-progress of labor and signs of fetal distress. As per institutional protocol, a pre-operative COVID-19 RT PCR from nasopharynx was sent. A term healthy female baby, weighing $2.865 \mathrm{~kg}$ was delivered and roomed in with the mother. COVID-19 RT PCR of the mother was tracked and found to be positive. Baby's COVID-19 RT PCR was sent at 18 hours of life and was negative. Mother wore mask and breastfed the baby. The dyad was asymptomatic and remained well on follow up. Since, COVID-19 infection was not suspected before $\mathrm{C}$ - section, health care workers (HCWs) were not wearing full personal protective equipment (PPE) and had to be quarantined.

Another extramural full term male neonate was delivered to a COVID-19 positive mother by C- section due to non-reactive non stress test and meconium stained liquor. Baby was born limp and required positive pressure ventilation for 2 minutes, followed by mechanical ventilation. Chest $\mathrm{X}$ ray showed bilateral heterogeneous opacities consistent with pneumonia and blood investigations revealed severe metabolic acidosis, thrombocytopenia (platelet count $18,000 \mathrm{~mm}^{3}$ ), while blood cultures were sterile. Baby developed shock in next few hours requiring inotropes which were gradually tapered over 2-3 days and baby was extubated on day 3 of life. On day 6 , baby developed seizures, was loaded with phenobarbitone and CT brain was done which was suggestive of hypoxic brain injury and suspicion of dural sinus thrombosis. Baby was referred to us for further management on day 8 of life. Neurological examination revealed reduced truncal and limb tone (lower limb > upper limb) and depressed neonatal reflexes. MRI brain with venography showed areas of restricted diffusion in periventricular deep white matter of bilateral frontal and parietal lobes, and subdural hemorrhage (Figure1). Nasopharyngeal swab for COVID19 RT PCR sent at day 3, 5 and 8 were negative. Baby was discharged on day 12 of life on direct feeds and anticonvulsants. 
The current pandemic of SARS-CoV-2 has most likely emerged from an animal source, probably bats along with an amplifying host. Human to human transmission occurs through airborne droplets and close personal contact. Nevertheless, considerable uncertainty exists regarding the potential vertical transmission of SARS-CoV-2 and its clinical manifestations in neonates. No evidence of transplacental transmission was seen in four recent published case series. [1-4] Wang $S$ reported a neonate who tested COVID-19 positive at 36 hours of life, born to a woman with SARS-CoV-2 infection. [5] Similarly, Zeng L et al in a case series of 33 neonates born to mothers with COVID-19 pneumonia, reported 3 neonates to be positive for SARS-CoV-2 on day 2 of life. [6] Albeit, COVID-19 tests in both these reports were performed following a variable period after birth, the possibility of vertical transmission cannot be ruled out. Nasopharyngeal swab for SARS-CoV-2 was negative in both our cases and is consistent with most reports. Amniotic fluid and breast milk tested negative in all these studies, but could not be done in our cases.

Second neonate in our case series suffered from catastrophic sequelae of hypoxic ischemic encephalopathy (HIE) with meconium aspiration pneumonia resulting in respiratory failure, shock and intracranial hemorrhage. A similar case of birth asphyxia was reported by Zeng et al in a preterm neonate born to COVID-19 positive mother. [6] Baby had pneumonia at birth, was positive of SARS-CoV-2 infection on day 2 and 4 of life and had concomitant Enterobacter sepsis. In both the cases, it remains unknown whether events could be attributed to COVID-19 positive status of the mother or these are manifestation of neonatal disease such as HIE with meconium aspiration syndrome or respiratory distress syndrome or sepsis. Further research is warranted to determine association of COVID 19 infection in pregnancy and neonatal outcomes.

SARS-CoV-2 pandemic appears to be long and protracted with likelihood to last for the next few months or even a couple of years. [7] HCWs remain to be at a constant risk of exposure. In a resource limited country like ours, testing all pregnant women for SARS-CoV-2 or wearing PPE in each delivery may not be feasible and will amount to wastage of resources. So, protocols need to be set to protect HCWs from exposure to asymptomatic carriers who are not tested for COVID-19 before delivery. Considering present scenario, in addition to universal precaution, use of N-95 mask should probably become a norm for all HCWs attending a delivery or C-section. Personnel attending a delivery or section should be minimized to conserve PPE. HCWs if unprotected and exposed to confirmed positive cases should be quarantined.

\section{Declarations}

Consent for patient 1 was not taken as anonymity was maintained and no picture or figure is being shared. Consent for patient 2 was taken as the figure of MRI Brain; an essential part of the manuscript is being shared.

\section{References}


1. Chen H, Guo J, Wang C, Luo F, Yu X, Zhang W, et al. Clinical characteristics and intrauterine vertical transmission potential of COVID-19 infection in nine pregnant women: a retrospective review of medical records. Lancet. 2020;395:809-15.

2. Liu W, Wang J, Li W,Zhou Z, Liu S, Rong Z, et al. Clinical characteristics of 19 neonates born to mothers with COVID-19. Front Med. 2020;13:1-6.

3. Wang X, Zhou Z,Zhang J, Zhu F, Tang Y, Shen X.A case of 2019 Novel Coronavirus in a pregnant woman with preterm delivery. Clin Infect Dis. 2020 Feb 28.doi: 10.1093/cid/ciaa200. [Epub ahead of print]

4. Zhu H, Wang L, Fang C, Peng S, Zhang L, Chang G, et al. Clinical analysis of 10 neonates born to mothers with 2019-nCoV pneumonia. Transl Pediatr.2020;9:51-60.

5. Wang S, Guo L, Chen L, Liu W, Cao Y, Zhang J et al. A case report of neonatal COVID-19 infection in China. Clin Infect Dis. 2020 Mar 12. doi: 10.1093/cid/ciaa225. [Epub ahead of print]

6. Zeng L, Xia S, Yuan W, Yan K,Xiao F, Shao J, et al. Neonatal Early-Onset Infection With SARS-CoV-2 in 33 Neonates Born to Mothers With COVID-19 in Wuhan, China. JAMA Pediatr. 2020 march 26. [Epub ahead of print].

7. How will the coronavirus outbreak end? Available from: https://www.livescience.com. [Accessed on 23 April 2020].

\section{Figures}

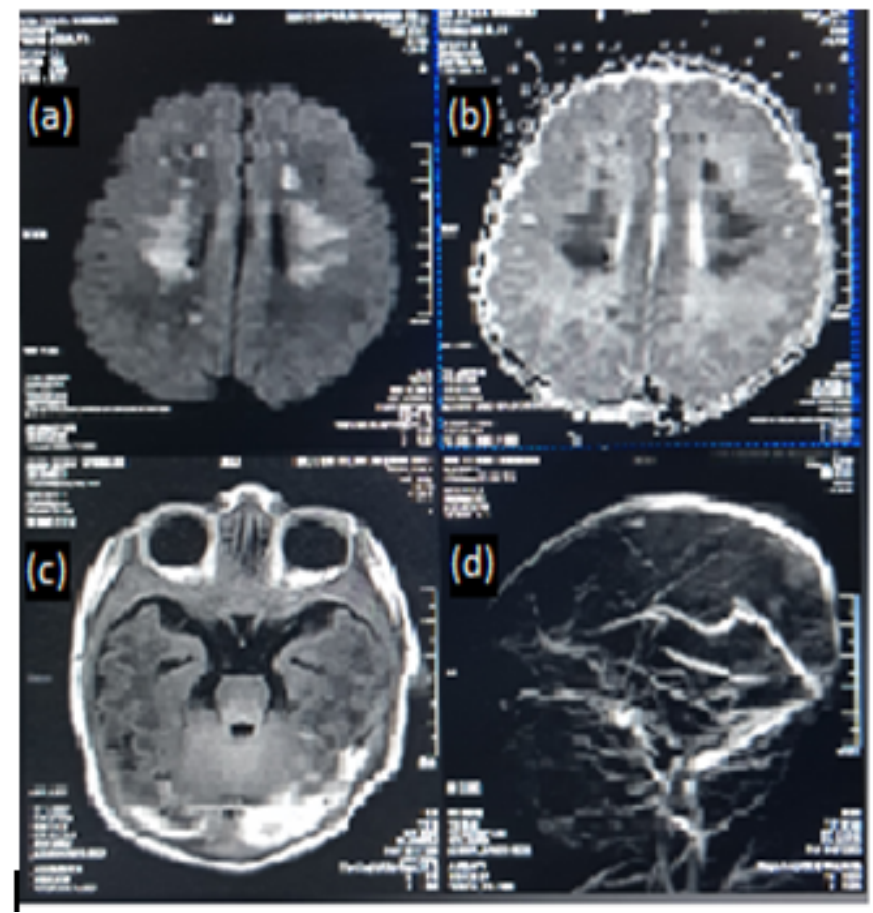

Figure 1 
$(a, b)$ Areas of restricted diffusion seen in periventricular deep white matter of the frontal and parietal lobes bilaterllay in DWI and SWI sequences respectively, consistent with hypoxic brain insult. (c) Subdural hemorrhage seen along posterior high parietal and occipital convexities extending along tentorium cerebelli and into the posterior fossa. (d) MR venography reveals no evidence of venous sinus thrombosis. 